\title{
Retrospective study of risk factors for colorectal adenomas and non-adenomatous polyps
}

\author{
Guanqun Chao", Yue Zhu", Lizheng Fang \\ Department of General Practice, Sir Run Run Shaw Hospital, Hangzhou 310016, China \\ Contributions: (I) Conception and design: G Chao; (II) Administrative support: L Fang; (III) Provision of study materials or patients: G Chao; (IV) \\ Collection and assembly of data: c; (V) Data analysis and interpretation: Yue Zhu; (VI) Manuscript writing: All authors; (VII) Final approval of \\ manuscript: All authors. \\ "These authors contributed equally to this work. \\ Correspondence to: Dr. Lizheng Fang. Department of General Practice, Sir Run Run Shaw Hospital, Hangzhou 310016, China. \\ Email: 3197056@zju.edu.cn.
}

\begin{abstract}
Background: To clarify the risk factors of colorectal adenomas and non-adenomatous polyps by retrospective analysis.

Methods: Data were obtained from subjects who underwent health examination in the Health Promotion Centre of Sir Run Run Shaw Hospital of Zhejiang University from January 2016 to December 2018, and all of them completed colonoscopies. Statistical analysis was performed using R software.

Results: A total of 721 subjects were included in the analysis, of whom 58 (8.0\%) were diagnosed as nonadenomatous polyps, $115(16.0 \%)$ as adenomas, $3(0.4 \%)$ as neuroendocrine neoplasms or adenocarcinomas. The gender ratio and prevalence of fatty liver in the non-adenomatous polyp group were different from that in the control group, and age, body mass index (BMI), waist circumference, blood glucose, serum uric acid, triglyceride, alanine transaminase and creatinine levels were higher, while the level of high-density lipoprotein was lower than control group $(\mathrm{P}<0.05)$. The sex ratio in the adenoma group was different from that in the control group, age, BMI, waist circumference, glycosylated hemoglobin, serum uric acid, triglyceride and creatinine levels were higher $(\mathrm{P}<0.05)$. Age was a risk factor for non-adenomatous polyps. Male and age were risk factors for adenomas.

Conclusions: Age, body weight, waist circumference, blood lipid and blood glucose are related to the high incidence of colorectal adenomas and non-adenomatous polyps; fatty liver is related to the high incidence of colorectal non-adenomatous polyps; age is a risk factor for non-adenomatous polyps; age and male are risk factors for colorectal adenomas.
\end{abstract}

Keywords: Colorectal adenoma (CRC); non-adenomatous polyp; fatty liver; risk factor

Submitted Sep 14, 2019. Accepted for publication Dec 20, 2019.

doi: $10.21037 /$ tcr.2020.01.69

View this article at: http://dx.doi.org/10.21037/tcr.2020.01.69

\section{Introduction}

Colorectal cancer (CRC) is the second most common malignancy in the world with a high mortality rate (1). Multiple guidelines indicate that people over age 50 with no symptoms should be screened for colon cancer $(2,3)$. Due to mutations in oncogenes and tumor suppressor genes, most CRCs originate from benign adenomas and develop into malignant tumors after more than 10 years (4). Colorectal adenomas and other types of proliferative polyps are considered precancerous lesions in CRC (5). In recent decades, the incidence of CRC is on the rise worldwide (6). Therefore, how to detect and treat the precancerous lesions of CRC early is an important clinical problem that needs to be solved.

The study found that age, family history, gender, race, 


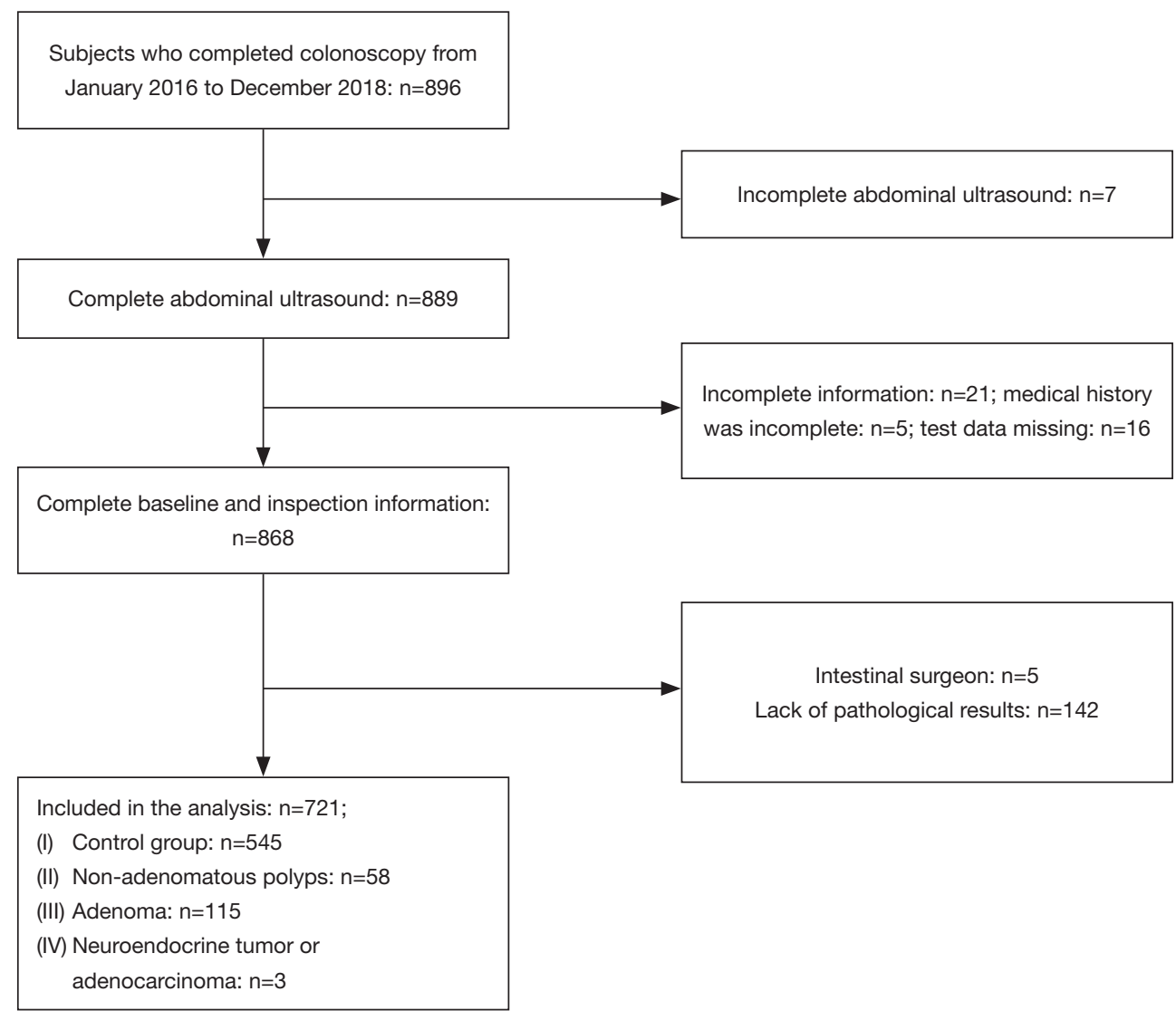

Figure 1 Subjects' inclusion process.

smoking and other factors were associated with CRC (7). The risk of CRC was significantly higher in diabetics than in other cancers (8). The increased concentration of serum insulin and insulin-like growth factor in patients with insulin resistance may promote the growth of precancerous lesions in CRC, thus increasing the possibility of malignant tumor (9). Insulin can stimulate colorectal cell proliferation and increase the biological activity of insulin-like growth factor-1 (IGF-1), thereby promoting cell mitosis and antiapoptosis (10). Recent studies suggest that the presence and severity of nonalcoholic fatty liver disease may be associated with the risk of CRC or adenoma (11). Nonalcoholic fatty liver disease with advanced fibrosis may be a risk factor for colorectal adenomas compared with nonalcoholic fatty liver disease without fibrosis (12). Adenoma and advanced neoplasia are associated with age, with twice as many cases occurring over age 45 (13). We will further clarify the risk factors of colorectal adenomas and hyperplastic polyps by retrospective analysis of the data of physical examination population, and verify the relationship between non- alcoholic fatty liver disease (NAFLD) and colorectal lesions.

\section{Methods}

\section{Data and methods}

Data were obtained from subjects who underwent health examination in the Health Promotion Centre of Sir Run Run Shaw Hospital of Zhejiang University from January 2016 to December 2018, and all of them completed colonoscopies (Figure 1). Exclusion criteria: (I) failed to complete the liver B-ultrasound examination; (II) lack of baseline information (incomplete history or partial test data); (III) patients after intestinal surgery; (IV) polyps and masses found in colonoscopy need pathological biopsy clear, but the patient refused or pathological biopsy data missing.

\section{Colonoscopy and biopsy}

All selected subjects were examined by an experienced 
colonoscopist using a video colonoscopy. Participants with colonic polyps were recommended for endoscopic polypectomy and biopsy. Biopsy specimens were examined by an experienced pathologist.

\section{Physical examination data acquisition}

When height and weight were measured, the subjects wore light clothes and no shoes. body mass index (BMI) is obtained by dividing weight $(\mathrm{kg})$ by height $(\mathrm{m})$ squared. Waist circumference is the length of one circle around the umbilical level. During the blood pressure measurement, the patient sat quietly for 10 minutes before the blood pressure measurement, and the systolic and diastolic blood pressure of the right upper limb were measured. Blood biochemical indicators, such as total cholesterol (TC), triglyceride (TG), low-density lipoprotein cholesterol (LDL-c), high-density lipoprotein cholesterol (HDL-c), glycosylated hemoglobin (HbA1c), alanine aminotransferase (ALT), cereal straw aminotransferase (AST), and glutamyl transpeptidase (GGT), were all completed by the hospital laboratory.

\section{Diagnosis and criteria of fatty liver}

Abdominal ultrasound was performed on all selected subjects. Abdominal ultrasonography was performed by experienced doctors, and the diagnosis of fatty liver was based on the guidelines for the prevention and treatment of fatty liver disease (2018 edition): B-ultrasonography was the first choice for the imaging diagnosis of fatty liver.

\section{Data analysis}

The data were analyzed by $\mathrm{R}$ software (version 3.5.1). Measurement data of normal distribution were described in the form of mean standard deviation $(\bar{x} \pm s)$, measurement data of non-normal distribution were described in the form of median (quartile), and counting data were described in the form of percentage. Student t-test was used for the mean value comparison of measurement data between the two groups of normal distribution, Wilcoxon symbolic rank test was used for the mean value comparison of measurement data between the two groups of normal distribution, and chi-square test was used for the comparison of counting data. Multivariate logistic regression analysis was performed to calculate the ratio (OR value) of age, gender, blood pressure, blood lipid and other factors. The statistical analysis was performed on both sides. When the $\mathrm{P}$ value was less than 0.05 , it was considered statistically significant.

\section{Results}

\section{Demographic and laboratory variables of the studied subjects}

Finally, 721 subjects were included in the analysis, of whom $58(8.0 \%)$ were diagnosed as non-adenomatous polyps, $115(16.0 \%)$ as adenomas, $3(0.4 \%)$ as neuroendocrine neoplasms or adenocarcinomas, and the remaining 545 $(75.6 \%)$ were classified as controls. The gender ratio and prevalence of fatty liver in the non-adenomatous polyp group were different from that in the control group, and the age, BMI, waist circumference, blood glucose, serum uric acid, triglyceride, alanine transaminase and creatinine levels were higher than those in the control group, while the levels of high-density lipoprotein were lower than those in the control group, with statistically significant differences $(\mathrm{P}<0.05)$. The sex ratio in the adenoma group was different from that in the control group, with statistically significant differences in age, BMI, waist circumference, glycosylated hemoglobin, serum uric acid, triglyceride and creatinine levels $(\mathrm{P}<0.05)$. Waist in the group of adenoma and the adenomatous polyp have differences, the age, BMI, blood pressure, fasting glucose, glycosylated hemoglobin, serum uric acid, triglyceride, total cholesterol, low density lipoprotein cholesterol, high-density lipoprotein cholesterol, alanine aminotransferase, aspartate aminotransferase, urea nitrogen, creatinine and so on had no statistically significant differences (Table 1).

\section{Logistic regression analysis of risk factors}

In the logistic regression model, male gender, age, BMI, systolic blood pressure, diastolic blood pressure, waist circumference, blood glucose, serum uric acid, triglyceride, total cholesterol, high-density lipoprotein, low-density lipoprotein and liver and kidney function were included in the analysis. Univariate regression analysis showed that gender, age, BMI, abdominal circumference, fasting blood glucose, uric acid, triglyceride level, high-density lipoprotein cholesterol level and fatty liver were risk factors for nonadenomatous polyps. After adjustment of the multivariate model, only age was a risk factor for non-adenomatous polyps. Similarly, univariate regression analysis showed that gender, age, BMI, abdominal circumference, blood 
Table 1 Demographic and laboratory analysis of adenoma and non-adenomatous polyp groups

\begin{tabular}{|c|c|c|c|c|c|c|}
\hline Factor & Control group $(n=545)$ & Non-adenomatous polyp $(n=58)$ & Adenoma $(n=115)$ & ${ }^{\mathrm{a}} \mathrm{P}$ & ${ }^{\mathrm{b}} \mathrm{P}$ & ${ }^{\mathrm{c}} \mathrm{P}$ \\
\hline Age, years & $48.8 \pm 8.45$ & $52.0 \pm 5.87$ & $51.1 \pm 7.66$ & $<0.001$ & 0.032 & 0.395 \\
\hline BMI, $\mathrm{kg} / \mathrm{m}^{2}$ & $24.4 \pm 3.15$ & $26.1 \pm 3.37$ & $25.2 \pm 2.97$ & $<0.001$ & 0.006 & 0.096 \\
\hline \multicolumn{7}{|l|}{$\mathrm{BP}, \mathrm{mmHg}$} \\
\hline DBP & $73.5 \pm 11.0$ & $75.6 \pm 8.93$ & $75.1 \pm 10.2$ & 0.105 & 0.143 & 0.733 \\
\hline WC, $\mathrm{cm}$ & $85.8 \pm 9.80$ & $92.3 \pm 10.3$ & $88.4 \pm 9.41$ & $<0.001$ & 0.009 & 0.0157 \\
\hline $\mathrm{FBG}^{*}, \mathrm{mmol} / \mathrm{L}$ & $5.12(3.60,15.8)$ & $5.37(4.20,13.8)$ & $5.32(4.00,11.1)$ & 0.013 & $<0.001$ & 0.879 \\
\hline $\mathrm{UA}, \mathrm{mg} / \mathrm{dL}$ & $352 \pm 90.7$ & $381 \pm 90.1$ & $383 \pm 88.0$ & 0.020 & $<0.001$ & 0.882 \\
\hline $\mathrm{HDL}, \mathrm{mg} / \mathrm{dL}$ & $1.16 \pm 0.294$ & $1.02 \pm 0.262$ & $1.10 \pm 0.290$ & $<0.001$ & 0.070 & 0.069 \\
\hline $\mathrm{LDL}, \mathrm{mg} / \mathrm{dL}$ & $2.82 \pm 0.762$ & $2.66 \pm 0.749$ & $2.80 \pm 0.728$ & 0.125 & 0.772 & 0.247 \\
\hline $\mathrm{ALT}^{\star}, \mathrm{IU} / \mathrm{L}$ & $21.0(5.00,181)$ & $27.5(7.00,86.0)$ & $23.0(9.00,79.0)$ & 0.005 & 0.133 & 0.113 \\
\hline AST, IU/L & $20.0(9.00,264)$ & $23.0(12.0,52.0)$ & $21.0(11.0,82.0)$ & 0.011 & 0.090 & 0.294 \\
\hline Fatty liver disease & 212 (38.9\%) & 33 (56.9\%) & $48(41.7 \%)$ & 0.001 & 0.645 & 0.085 \\
\hline
\end{tabular}

*, it makes sense between the groups. ${ }^{\text {a }}$, two-sided $\mathrm{P}$ values for the difference between the non-adenomatous polyp group and control group; ${ }^{b} \mathrm{P}$, two-sided $\mathrm{P}$ values for the difference between the adenoma group and control group; ${ }^{\mathrm{C}} \mathrm{P}$, two-sided $\mathrm{P}$ values for the difference between the adenoma group and non-adenomatous polyp group. BP, blood pressure; SBP, systolic blood pressure; DBP, diastolic blood pressure; WC, Waist circumference; FBG, fast blood glucose; SUA, serum Uric acid; TC, total cholesterol; TG, triglycerides; LDL, low density lipoprotein; HDL, high density lipoprotein; ALT, alanine aminotransferase; AST, aspartate aminotransferase; BUN, blood urea nitrogen.

glucose, uric acid, triglyceride level, creatinine, and urea nitrogen were risk factors for adenomas. After adjustment of the multivariate model, male and age were risk factors for adenomas (Table 2).

\section{Discussion}

CRC is considered a major public health problem due to its high morbidity and mortality. Recent studies have found that CRC is also increasing in morbidity and mortality among adolescents (14). Because CRC screening rate increased, adenoma early resection, in recent years, adult CRC mortality has been reduced (15). Molecular evidence suggests that CRC may be caused by mixed polyps, in addition to cytologic dysplasia (16). A prospective study found that none of the carriers of polypos-related mutations developed colorectal polyps (17). This shows, although the happening of CRC thinks to be concerned with heredity, but gene is not the main reason that appears CRC pathological change.

Our study analyzed patients who completed colonoscopies between 2016 and 2018. In the 721 subjects, $58(8.0 \%)$ were diagnosed as non-adenomatous polyps, $115(16.0 \%)$ as adenomas, $3(0.4 \%)$ as neuroendocrine neoplasms or adenocarcinomas. Compared with the control group, age, BMI, waist circumference, blood glucose, serum uric acid, triglyceride of non-adenomatous polyps group and adenoma group were higher, and HDL was lower in non-adenomatous polyps group, AST was higher in nonadenomatous polyps group as well. Previous studies have shown that metabolic syndrome is associated with CRC and colorectal polyps (18). Studies on colorectal hyperplastic polyps suggest that metabolic syndrome is a high-risk factor (19). Our study showed that blood glucose, serum uric acid and triglyceride levels increased significantly in patients with colonic adenoma and other polyps, this is consistent with the research of other scholars. Current studies suggest that the mechanism may be related to 
Table 2 Risk factors analysis of adenoma group and non-adenomatous polyp group

\begin{tabular}{|c|c|c|c|c|c|c|}
\hline Factor & \multicolumn{3}{|c|}{ Crude } & \multicolumn{3}{|c|}{ Adjusted* } \\
\hline \multicolumn{7}{|c|}{ Non-adenomatous polyp } \\
\hline Male gender & 3.548 & $1.790-7.858$ & $<0.001^{*}$ & 2.416 & $1.022-6.123$ & 0.051 \\
\hline Age & 1.047 & $1.013-1.082$ & $0.006^{*}$ & 1.045 & $1.009-1.083$ & $0.015^{*}$ \\
\hline DBP & 1.017 & $0.993-1.043$ & 0.166 & - & - & - \\
\hline WC & 1.070 & $1.040-1.103$ & $<0.001^{*}$ & 1.026 & $1.539-1.668$ & 0.262 \\
\hline FBG & 1.287 & $1.096-1.504$ & $0.001^{*}$ & 1.133 & $1.645-1.769$ & 0.174 \\
\hline SUA & 1.003 & $1.000-1.006$ & $0.019^{*}$ & 0.999 & $0.099-1.003$ & 0.520 \\
\hline HDL & 0.142 & $0.043-0.430$ & $<0.001^{*}$ & 0.354 & $0.908-1.258$ & 0.121 \\
\hline LDL & 0.751 & $0.517-1.079$ & 0.127 & - & - & - \\
\hline ALT & 1.011 & $0.998-1.023$ & 0.076 & - & - & - \\
\hline AST & 1.006 & $0.985-1.022$ & 0.447 & - & - & - \\
\hline FLD & 2.073 & $1.203-3.615$ & $0.009^{*}$ & 0.824 & $0.401-1.686$ & 0.595 \\
\hline \multicolumn{7}{|l|}{ Adenoma } \\
\hline Male gender & 2.345 & $1.479-3.840$ & $<0.001^{*}$ & 2.042 & $1.018-4.179$ & $0.047^{\star}$ \\
\hline Age & 1.033 & $1.008-1.058$ & $0.009^{*}$ & 1.036 & $1.010-1.063$ & $0.007^{\star}$ \\
\hline SUA & 1.004 & $1.002-1.006$ & $<0.001^{*}$ & 1.002 & $0.999-1.005$ & 0.197 \\
\hline TG & 1.135 & $1.031-1.278$ & $0.023^{*}$ & 1.085 & $0.986-1.222$ & 0.131 \\
\hline $\mathrm{TC}$ & 1.179 & $0.955-1.453$ & 0.123 & - & - & - \\
\hline HDL & 0.507 & $0.237-1.040$ & 0.717 & - & - & - \\
\hline LDL & 0.962 & $0.735-1.255$ & 0.777 & - & - & - \\
\hline ALT & 1.003 & $0.991-1.013$ & 0.651 & - & - & - \\
\hline AST & 1.007 & 0.992-1.022 & 0.299 & - & - & - \\
\hline $\mathrm{CR}$ & 1.015 & $1.002-1.029$ & $0.027^{\star}$ & 0.999 & $0.979-1.019$ & 0.908 \\
\hline BUN & 1.124 & 0.918-1.372 & $<0.001^{*}$ & 0.940 & $0.746-1.178$ & 0.593 \\
\hline FLD & 1.125 & $0.745-1.690$ & 0.571 & - & - & - \\
\hline
\end{tabular}

Crude means only single factor regression analysis without adjusting age, gender and other factors. *, it makes sense between the groups. Gender, age, BMI, central obesity, diabetes, different levels of uric acid, high TC, high TG, high LDL-C, low HDL-c, and abnormal liver function were Adjusted. OR, odds ratio; BMI, body mass index; SUA, serum uric acid; TC, total cholesterol; HDL, high-density lipoproteins; LDL, low-density lipoproteins; LF, liver function. 
insulin resistance in patients with metabolic syndrome (20). The research showed that older age $(\geq 60)$, male sex, and higher BMI $(\geq 25)$ were associated with the development of colorectal adenoma (21). Previous studies have suggested that the presence of metabolic syndrome, or an increase in BMI, is associated with the risk of colorectal adenoma and CRC (22). Our research showed that the higher BMI and higher waist circumference in non-adenomatous polyps group and adenoma group was similar. We further performed a regression analysis of risk factors for colorectal adenomas and non-adenomatous polyps. Age and male gender were the risk factors of colorectal adenomas, and only age was the risk factor of non-adenomatous polyps. There are currently several guidelines on CRC screening published in many countries or organizations, and the guidelines clearly indicate the correlation between age and colon cancer screening, which is consistent with our study that age is an important risk factor.

NAFLD is a kind of liver disease without viral hepatitis, drug-induced liver injury, excessive alcohol consumption, hereditary disorders and so on, as a result of its high prevalence it becomes an important public health issue (23). A large Korean population study found a direct association between nonalcoholic fatty liver disease and colorectal adenomas (24). Scholars have pointed out that the formation of colon tumors is related to the severity of fatty liver, but the correlation is not significant under the interference of other factors (25). Non-alcoholic steatohepatitis has also been shown to be associated with a higher incidence of CRC and CRC progression (26). Although it is currently believed that non-alcoholic fatty liver may be related to the occurrence of CRC and colorectal adenoma, there are conflicting opinions, and the causes and mechanisms of its high incidence are still unclear. In this study, it was found that the incidence of fatty liver was significantly increased in the colorectal non-adenomatous polyp group, while there was no significant difference in the adenoma group. At the same time, fatty liver was not considered as a risk factor for colorectal adenomas and non-adenomatous polyps after multivariate regression analysis. The increased incidence of fatty liver indicates that fatty liver is related to the occurrence of colorectal non-adenomatous polyps, which is consistent with the studies of some scholars. However, our analysis failed to prove that fatty liver could be a risk factor for colorectal adenomas and non-adenomatous polyps, for the following reasons: (I) the sample size was limited and there was a large gap between the groups; (II) the selected subjects were located in southeast China, and their living habits, genetic factors, diet, soil and water were different from other countries; (III) similar to previous studies, there were other factors that interfered.

Insulin resistance is believed to be an important reason for the increased incidence of CRC in patients with metabolic syndrome (27). In addition, high NLR was associated with increased colorectal adenomatous polyps (28). Thus, inflammatory cytokines such as tumor necrosis factor- $\alpha$ and interleukin- 6 and other proinflammatory cytokines play a role in colorectal adenomas and carcinomas (29). In addition, nonalcoholic fatty liver disease is thought to be mostly insulin resistant (30). As a result, patients with nonalcoholic fatty liver disease have a higher incidence of colorectal adenomas and cancers. Our study failed to find a correlation between fatty liver and colorectal adenoma, which may be related to the presence of insulin resistance and chronic inflammation in the patients. Our study found that age, weight, waist circumference, blood lipids, blood glucose and other factors were related to colorectal adenomas and non-adenomatous polyps. The incidence of fatty liver in patients with nonadenomatous polyps was significantly increased, while age was a risk factor for non-adenomatous polyps, and age and male were risk factors for colorectal adenomas. To sum up, we suggest that the patients with overweight, abnormal metabolic indicators and fatty liver should pay attention to colonoscopy examination, especially older men, and should have colonoscopy screening regularly.

\section{Conclusions}

Age, body weight, waist circumference, blood lipid and blood glucose are related to the high incidence of colorectal adenomas and non-adenomatous polyps; fatty liver is related to the high incidence of colorectal non-adenomatous polyps; age is a risk factor for non-adenomatous polyps; age and male are risk factors for colorectal adenomas.

\section{Acknowledgments}

Funding: This research was supported by funding from Zhejiang Provincial Natural Science Foundation of China under Grant No. LY18H030001; the Medicine and Health Science and Technology Plan Projects in Zhejiang Province (2017KY413), Traditional Chinese Medicine Science and Technology Plan of Zhejiang Province (2017ZA089, 2016ZB071, 2015ZZ012, 2014ZA030); National Natural Science Foundation of China (81573760); Medical Health 
Platform Plan Projects of Zhejiang Province (2015RCA020); Zhejiang Provincial Natural Science Foundation of China (LY16H030010).

\section{Footnote}

Conflicts of Interest: All authors have completed the ICMJE uniform disclosure form (available at http://dx.doi. org/10.21037/tcr.2020.01.69). The authors have no conflicts of interest to declare.

Ethical Statement: The authors are accountable for all aspects of the work in ensuring that questions related to the accuracy or integrity of any part of the work are appropriately investigated and resolved. The study was conducted in accordance with the Declaration of Helsinki (as revised in 2013). This study was approved by the ethics committee of Zhejiang University and oral informed consent was obtained from all patients.

Open Access Statement: This is an Open Access article distributed in accordance with the Creative Commons Attribution-NonCommercial-NoDerivs 4.0 International License (CC BY-NC-ND 4.0), which permits the noncommercial replication and distribution of the article with the strict proviso that no changes or edits are made and the original work is properly cited (including links to both the formal publication through the relevant DOI and the license). See: https://creativecommons.org/licenses/by-nc-nd/4.0/.

\section{References}

1. Goshayeshi L, Pourahmadi A, Ghayour-Mobarhan M, et al. Colorectal cancer risk factors in north-eastern Iran: A retrospective cross-sectional study based on geographical information systems, spatial autocorrelation and regression analysis. Geospat Health 2019. doi: 10.4081/gh.2019.793.

2. Monahan KJ, Bradshaw N, Dolwani S, et al. Guidelines for the management of hereditary colorectal cancer from the British Society of Gastroenterology (BSG)/Association of Coloproctology of Great Britain and Ireland (ACPGBI)/ United Kingdom Cancer Genetics Group (UKCGG). Gut 2019. [Epub ahead of print]

3. Bisschops R, East JE, Hassan C, et al. Advanced imaging for detection and differentiation of colorectal neoplasia: European Society of Gastrointestinal Endoscopy (ESGE) Guideline - Update 2019. Endoscopy 2019;51:1155-79.

4. Markowitz SD, Bertagnolli MM. Molecular origins of cancer: Molecular basis of colorectal cancer. N Engl J Med 2009;361:2449-60.

5. Sanyaolu LN, Oakley NJ, Nurmatov U, et al. Antibiotic exposure and the risk of colorectal adenoma and carcinoma: systematic review and meta-analysis of observational studies. Colorectal Dis 2019. [Epub ahead of print].

6. Arnold M, Sierra MS, Laversanne M, et al. Global patterns and trends in colorectal cancer incidence and mortality. Gut 2017;66:683-91.

7. Kim BC, Shin A, Hong CW, et al. Association of colorectal adenoma with components of metabolic syndrome. Cancer Causes Control 2012;23:727-35.

8. Voutsadakis IA. Obesity and diabetes as prognostic factors in patients with colorectal cancer. Diabetes Metab Syndr 2017;11 Suppl 1:S109-14.

9. Aslan A, Erdem H, Celik MA, et al. Investigation of InsulinLike Growth Factor-1 (IGF-1), P53, and Wilms' Tumor 1 (WT1) Expression Levels in the Colon Polyp Subtypes in Colon Cancer. Med Sci Monit 2019;25:5510-7.

10. Hosseini SA, Zand H, Cheraghpour M. The Influence of Curcumin on the Downregulation of MYC, Insulin and IGF-1 Receptors: A possible Mechanism Underlying the Anti-Growth and Anti-Migration in Chemoresistant Colorectal Cancer Cells. Medicina (Kaunas) 2019. doi: 10.3390/medicina55040090.

11. Chen J, Bian D, Zang S, et al. The association between nonalcoholic fatty liver disease and risk of colorectal adenoma and cancer incident and recurrence: a metaanalysis of observational studies. Expert Rev Gastroenterol Hepatol 2019;13:385-95.

12. Kim MC, Park JG, Jang BI, et al. Liver fibrosis is associated with risk for colorectal adenoma in patients with nonalcoholic fatty liver disease. Medicine (Baltimore) 2019;98:e14139.

13. Karsenti D, Tharsis G, Burtin P, et al. Adenoma and advanced neoplasia detection rates increase from 45 years of age. World J Gastroenterol 2019;25:447-56.

14. Bailey CE, Hu CY, You YN, et al. Increasing disparities in the age-related incidences of colon and rectal cancers in the United States, 1975-2010. JAMA Surg 2015;150:17-22.

15. Burke C, Kaul V, Pohl H. Polyp Resection and Removal Procedures: Insights From the 2017 Digestive Disease Week. Gastroenterol Hepatol (N Y) 2017;13:1-24.

16. Cappellesso R, Lo Mele M, Munari G, et al. Molecular characterization of "sessile serrated" adenoma to carcinoma transition in six early colorectal cancers. Pathol 
Res Pract 2019;215:957-62.

17. El Hachem N, Abadie C, Longy M, et al. Endoscopic Phenotype of Monoallelic Carriers of MUTYH Gene Mutations in the Family of Polyposis Patients: A Prospective Study. Dis Colon Rectum 2019;62:470-5.

18. Esposito K, Capuano A, Giugliano D. Metabolic syndrome and cancer: holistic or reductionist?Endocrine 2014;45:362-4.

19. Chen FP, Wang HM, Chiang FF, et al. The metabolic syndrome is associated with an increased risk of colorectal polyps independent of plasma homocysteine. Ann Nutr Metab 2014;64:106-12.

20. Tae CH, Kim SE, Jung SA, et al. Involvement of adiponectin in early stage of colorectal carcinogenesis. BMC Cancer 2014;14:811.

21. Ze EY, Kim BJ, Jun DH, et al. The Fatty Liver Index: A Simple and Accurate Predictor of Colorectal Adenoma in an Average-Risk Population. Dis Colon Rectum 2018;61:36-42.

22. Liu CS, Hsu HS, Li CI, et al. Central obesity and atherogenic dyslipidemia in metabolic syndrome are associated with increased risk for colorectal adenoma in a Chinese population. BMC Gastroenterol 2010;10:51.

23. Chen Z, Liu F, Zheng N, et al. Wuzhi capsule (Schisandra Sphenanthera extract) attenuates liver steatosis and

Cite this article as: Chao G, Zhu Y, Fang L. Retrospective study of risk factors for colorectal adenomas and nonadenomatous polyps. Transl Cancer Res 2020;9(3):1670-1677. doi: $10.21037 /$ tcr.2020.01.69 inflammation during non-alcoholic fatty liver disease development. Biomed Pharmacother 2019;110:285-93.

24. Hwang ST, Cho YK, Park JH, et al. Relationship of nonalcoholic fatty liver disease to colorectal adenomatous polyps. J Gastroenterol Hepatol 2010;25:562-7.

25. Lee T, Yun KE, Chang Y, et al. Risk of Colorectal Neoplasia According to Fatty Liver Severity and Presence of Gall Bladder Polyps. Dig Dis Sci 2016;61:317-24.

26. Wong VW, Wong GL, Tsang SW, et al. High prevalence of colorectal neoplasm in patients with non-alcoholic steatohepatitis. Gut 2011;60:829-36.

27. Uyar GO, Sanlier N. Association of Adipokines and Insulin, Which Have a Role in Obesity, with Colorectal Cancer. Eurasian J Med 2019;51:191-5.

28. Kim JH, Cho KI, Kim YA, et al. Elevated Neutrophil-toLymphocyte Ratio in Metabolic Syndrome Is Associated with Increased Risk of Colorectal Adenoma. Metab Syndr Relat Disord 2017;15:393-9.

29. Szylberg $€$, Janiczek M, Popiel A, et al. Expression of COX-2, IL-1 $\beta$, TNF- $\alpha$ and IL-4 in epithelium of serrated adenoma, adenoma and hyperplastic polyp. Arch Med Sci 2016;12:172-8.

30. Kitade H, Chen G, Ni Y, et al. Nonalcoholic Fatty Liver Disease and Insulin Resistance: New Insights and Potential New Treatments. Nutrients 2017. doi: 10.3390/nu9040387. 